\title{
Zehn Jahre Journal für Verbraucherschutz und Lebensmittelsicherheit: gerne weiter so!
}

\author{
Saskia Dombrowski ${ }^{1}$
}

Published online: 26 July 2015

(C) Bundesamt für Verbraucherschutz und Lebensmittelsicherheit (BVL) 2015

Das Journal für Verbraucherschutz und Lebensmittelsicherheit (JVL) feiert sein zehnjähriges Bestehen und hat sich als innovatives Format rund um den Zuständigkeitsbereich des herausgebenden Bundesamtes für Verbraucherschutz und Lebensmittelsicherheit (BVL) etabliert. Rückblickend ist dieser Prozess schnell von statten gegangen und so freut sich die JVLRedaktion über die aktuelle Einschätzung des BVLPräsidenten, Dr. Helmut Tschiersky, in der er das JVL als eine Visitenkarte des BVL bezeichnet und der Zeitschrift ein „Weiter so!“ für die kommenden (zehn) Jahre auf den Weg gibt. Ganz im Sinne des Mottos „nicht älter, sondern besser werden“, möchten wir gerne noch eine Schippe drauflegen und freuen uns auf weitere Entwicklungen des JVL. Apropos: Das vollständige Interview mit Dr. Tschiersky finden Sie in der vorliegenden JVL-Ausgabe.

Von Beginn an hatte das JVL den hohen Anspruch an sich selbst, sowohl als ein Medium der behördlichen Kommunikation zu fungieren als auch den Anforderungen einer seriösen Wissenschaftskommunikation gerecht zu werden. Es entstand ein lebendiges Forum, das den Lesern komplexe Zusammenhänge aus der gesamten Vielfalt des gesundheitlichen Verbraucherschutzes - vom Acker bis zum Teller - differenziert und in wissenschaftlich hochwertiger Qualität zur Verfügung stellt.

Saskia Dombrowski

jvl@bvl.bund.de

$1 \quad$ Bundesamt für Verbraucherschutz und Lebensmittelsicherheit, Mauerstr. 39-42, 10117 Berlin, Germany
Im breiten und abwechslungsreichen Themenspektrum der Beiträge, die in den letzten Jahren im JVL erschienen sind, spiegeln sich erwartungsgemäß etliche wichtige Ereignisse und Entwicklungen dieses Zeitraums für den Verbraucherschutz wider. In über 40 Ausgaben widmeten sich mehr als 500 Beiträge diversen Aspekten des gesundheitlichen Verbraucherschutzes. Angefangen bei A wie Analytik bis W wie weltweite Warenströme wurden Artikel zu E-commerce, EHEC, Gentechnik, Klimawandel und Welternährung, Lebensmittelsicherheit, Neuromarketing, Neonikotinoide, Tierarzneimittel und Verbraucherforschung und vielem mehr veröffentlicht.

An dieser Stelle möchte ich die Gelegenheit nutzen und mich im Namen der JVL-Redaktion bei allen Wissenschaftlerinnen und Wissenschaftlern, die das JVL unterstützen, herzlich bedanken. Als Autorin oder Autor neuester Ergebnisse und Einschätzungen beziehungsweise als Gutachterin und Gutachter in der Bewertung von Einreichungen, leisten Sie einen essentiellen Beitrag zur Qualität der Zeitschrift. Bitte machen auch Sie unbedingt "weiter so".

Mit seinem Profil ist es dem JVL in recht kurzer Zeit gelungen, sich innerhalb der Zeitschriften der Kategorie Food Science and Technology zu etablieren. Der im Jahr 2009 von Thomson ISI (Institute for Scientific Information) erstmals ermittelte Impact Factor des JVL steigt stetig an und die steigende Zitationsrate ist ein Hinweis auf die Relevanz der Beiträge im JVL für die wissenschaftliche Gemeinschaft. Dies gelingt trotz der erwähnten dualen Zielsetzung der Zeitschrift, die eine alleinige Fokussierung auf die Veröffentlichung von für die Forschung relevanten Beiträgen weder erstrebenswert noch zielführend macht. 
Auch die Zahl der Einreichungen und die Verbreitung des Journals steigen weiterhin. Etwa ein Drittel der Beiträge, die als Peer-reviewed-Artikel im JVL eingehen, werden aktuell zur Publikation akzeptiert und erreichen online eine Leserschaft in etwa 7.500 Institutionen - vor allem in Deutschland und Europa, aber auch weltweit. Etwa 30.000 Mal werden jährlich JVL-Artikel heruntergeladen.

Die vorliegende Jubiläumsausgabe widmet sich dem „State of the art" verschiedener Zuständigkeiten des BVLs, dem Herausgebers des Geburtstagskindes. Ganz überwiegend sind es daher Mitarbeiterinnen und Mitarbeiter des BVL, die die Beiträge in diesem Heft verfasst haben und aktuelle Themenschwerpunkte der fünf Abteilungen des Bundesamtes präsentieren. Den Anfang macht ein Portrait der Gemeinsamen Expertenkommission des BVL und des Bundesinstituts für Arzneimittel und Medizinprodukte. Ziel dieser 2013 ins Leben gerufenen „Gemeinsamen Expertenkommission zur Einstufung von Borderline-Stoffen, die als Lebensmittel oder Lebensmittelzutat in den Verkehr gebracht werden“ ist es, auf Basis wissenschaftlicher Stellungnahmen (Management-)Empfehlungen zum Umgang mit einzelnen Stoffen oder Stoffgruppen zu erarbeiten. Drei weitere Beiträge kommen ebenfalls aus der Abteilung Lebensmittelsicherheit. Der Erste befasst sich mit Krisenmanagement und -übungen im gesundheitlichen Verbraucherschutz. Dargestellt wird die Bund-Länder-Vereinbarung zum Krisenmanagement, die im Nachgang zu den Geschehnissen rund um Dioxine in Futterfetten und -ölen und dem EHECKrankheitsausbruch im Jahr 2011 etabliert wurde. Im Zweiten wird das Zoonosen-Monitoring vorgestellt. Die erfassten Daten zum Vorkommen der wichtigsten Zoonoseerreger auf allen Stufen der Lebensmittelkette ermöglichen es, Rückschlüsse auf das Infektionsrisiko für Verbraucher durch den Verzehr von Lebensmitteln zu ziehen. Last but not least: Eine besondere Herausforderung ist zurzeit die Kontrolle des Onlinehandels verschiedener Waren - Kolleginnen und Kollegen der Bund-Länder-Kontrollstelle Internethandel / G@ZIELT erläutern Erfahrungen aus zwei Jahren Kontrolle.

Rund um Pflanzenschutzmittel (PSM) dreht es sich bei der Beschreibung des aktuellen Standes der Zulassung des Wirkstoffes Glyphosat sowie der Darstellung der Ergebnisse der PflanzenschutzKontrollprogramme. Im Rahmen von Handelskontrollen zur Überwachung der Verkehrsfähigkeit von PSM entnehmen die Pflanzenschutzdienste der Bundesländer Proben und prüfen die korrekte Zusammensetzung und die Einhaltung sonstiger gesetzlich festgelegte Bedingungen.
Ein Beitrag zur Tierarzneimittel (TAM)-Zulassung, einem weiteren Schwerpunkt des BVL, beschreibt das „Benchmarking of European Medicines Agencies“, das das BVL als eine der nationalen Arzneimittelbehörden, die für die Zulassung von TAM in der EU zuständig sind, im Jahr 2014 zum dritten Mal sehr erfolgreich durchlaufen hat. Ein anderer Artikel befasst sich mit einem Dauerbrenner auf dem Gebiet der TAM, den Antiobiotika, und erläutert die Gesetzgebung und Datenerfassung zu diesen Mitteln in Deutschland.

Den Themenkreis der Gentechnik eröffnet ein Beitrag mit Fokus auf das Influenzavirus, welches mit seinem auffällig wandlungsfähigen Genom eine besondere Herausforderung für die Risikobewertung und für die Auswahl angemessener Sicherheitsmaßnahmen beim Umgang mit Influenzaviren darstellt. Ein anderer Artikel beschreibt neue Techniken der Molekularbiologie, wie die RNAi-Technik und neue Möglichkeiten des Genome engineering. Konkret geht es um die Verwendung des CRISPR-Cas9Systems - eine möglicherweise nobelpreiswürdige Technik, deren Möglichkeiten noch nicht ausgeschöpft sind und die Bewertung von mit diesen Techniken veränderten Organismen herausfordern.

Eine Einschätzung der Erfordernisse, die sich aus dem globalen Handel mit Lebensmitteln an die Analytik stellen, eröffnet die Reihe der Beiträge aus der Abteilung Methodenstandardisierung, Referenzlabore und Antibiotikaresistenz des BVL. Weiterhin werden das System der Leistungsfähigkeitsprüfung von Untersuchungsmethoden der Lebensmittelkontrolle sowie die Erfassung von Daten aus Eignungsprüfungen erläutert und ihre Aussagekraft hinsichtlich einer angegebenen Messunsicherheit eingeschätzt. Zu guter Letzt stellt sich das Nationale Resistenzmonitoring tierpathogener Erreger (GERM-Vet) vor, in dem das BVL die Empfindlichkeit tierpathogener Bakterien gegenüber Antibiotika untersucht, um die aktuelle Resistenzsituation zu ermitteln.

„Risiken managen - Verbraucher schützen“ so lautet das Motto des BVL. Zu seinem zehnjährigen Jubiläum präsentiert das JVL ein weitgefächerten Kaleidoskop an Themen, das die Zuständigkeiten des BVL für den gesundheitlichen Verbraucherschutz widerspiegelt. Ein JVL-Maxime in diesem Kontext könnte heißen: „Zusammenhänge und Risiken vielseitig kommunizieren - Leser differenziert informieren“.

Ich wünsche Ihnen eine informative und interessante Lektüre. 